\title{
Time-Frequency Localization Optimization of PR FMT: Case of an Overlapping Factor Equal to 2
}

\author{
Didier Pinchon ${ }^{1}$ and Pierre Siohan ${ }^{2}$ \\ ${ }^{1}$ Institut de Mathématiques de Toulouse, Université Paul Sabatier , \\ Toulouse, France \\ ${ }^{2}$ Independent Researcher, Brittany, France
}

April 10, 2019

\begin{abstract}
In this paper, we analyze the perfect reconstruction property of Filtered MultiTone (FMT) systems with overlapping factor equal to 2 . If small overlapping factors are not common for FMT systems that originally targeted nearly perfect subchannels isolation, we show that they can provide pretty good results when the optimization is carried out with the Time-Frequency Localization (TFL) criterion. Furthermore, it appears that the TFL criterion can ease the treatment of the PR equations. In this respect, our study exhibits nearly optimal TFL results and also leads to a simple and interesting closed form expression for the PR FMT prototype filter coefficients. Numerical results and illustrations are also provided showing the good behavior of our proposed design method in terms of TFL performance and computational complexity.
\end{abstract}

Keyword: Compact Representation, FMT, OFDM, Overlapping Factor, Perfect Reconstruction, Root Raised Cosine, Time-Frequency Localization

\section{Introduction}

Various research tracks have been envisioned to determine the most appropriate waveforms for the future $5 \mathrm{G}$ communication systems. At the end, for compatibility reasons, simple variants around the 4G Orthogonal Frequency Division Multiplexing (OFDM) scheme could be preferred. In particular, the Weighted Overlap and Add (WOLA)-OFDM system [1], [2] has gained a large interest into the the $3 \mathrm{GPP}$ standardization community. Indeed, similarly to CP-OFDM, it guarantees low computational complexity and latency. The difference with CP-OFDM mainly comes from the introduction of soft edges at the borders of the rectangular OFDM window function. We have recently shown that, if these transitions are calculated using the discrete Raised Root Cosine (RRC) function in time, we thus called 
dual RRC (dRRC) [3], this waveform is nearly optimal when considering the Time-Frequency Localization (TFL) criterion.

Since the mid-nineties and a famous publication about Coded OFDM systems [4], the TFL criterion is considered as a highly relevant objective function for the design of multicarrier (MC) systems for transmission over time and frequency dispersive channels. Furthermore, as it naturally leads to compact waveforms in time and frequency, for a given transmission performance, the resulting computational complexity is relatively low. In [3], we provided Optimal TFL (OTFL) windows with duration limited, as also the case for WOLA-OFDM, to one CP-OFDM symbol, i.e. an overlapping factor equal to 1 . Such a short time window can be also considered as the prototype filter for Filtered MultiTone (FMT) systems satisfying the Perfect Reconstruction (PR) property. With this PR FMT scheme, differently from the original FMT system [5], there is no overlapping in time but in frequency. So this MC system also received other names as, for instance, Weighted Cyclic Prefix (WCP)-OFDM in [6] or Pulse-Shaped OFDM in [7]. Here, for simplicity, we keep the FMT denomination for all these types of MC systems in which waveform overlapping may occur in time and/or in frequency. As recalled in [7], even if the shortest windows are preferred for the enhanced mobile broadband (eMBB) service, given the large extent of other envisioned services in $5 \mathrm{G}$, the requirements may vary accordingly and waveform constraints too. For instance, massive Machine Type Communication (mMTC) does not necessarily require short packet transmission and then longer waveforms are of interest if they can bring some improvements. In this sense, in this paper, we propose a new FMT system with a prototype filter the length of which has a duration equal to two MC symbols,i.e. an overlapping factor equal to 2 .

Denoting by $M$ its number of subcarriers and $N$ its expansion/decimation factor, that means we focus on the design of a prototype filter with length $L=2 N$. We also constrain this FMT system to fulfill the PR property. As the Spectral Efficiency (SE) is a key parameter for transmission systems, this has resulted sometimes before to only consider a subset of $(M, N)$ parameters values for FMT designs. For instance in $[8,9,10]$, the authors focus on the case where $\Delta=\operatorname{gcd}(N, M)$ with $M=\Delta M_{0}, N=\Delta N_{0}$ and $N_{0}=M_{0}+1$. If this parameter setting can perfectly fit for some applications, as e.g. the DVB-T2 broadcasting standard [11] in which we have $M=32768$ and $N=33792$ for the $32 \mathrm{~K}$ mode, this is no longer true for many other important wired or wireless applications.

In [3] we have proposed a $N$-length FMT system that circumvents this limitation. In this paper our aim is to go a step further, proposing for $L=2 N$ PR FMT systems being, differently from [8], usable for all parameters values as long as $M<N \leq 2 M-1$. By the way, we propose an FMT alternative to nearly all existing systems initially built on a CPOFDM kernel, with $M$ samples useful duration and a $(N-M)$-CP length being less than $M$, as it is the case for many wired and wireless communication standards, cf. for instance, IEEE P1901 standard for Power Line Communication [12] and the LTE for mobile radio.

Our paper is organized as follows. In Section 2, we present a background concerning CP-OFDM and FMT systems, the PR property for FMT systems and the TFL criterion. In Section 3, we study the PR conditions for the particular case $L=2 N$ and we obtain an angular representation for a significant subset of PR filters capable of achieving the optimal TFL. This optimization process is greatly simplified by using a compact representation (CR) as studied in Section 4. A new family of PR filters of length $2 N$, for which we have derived a 
closed form expression and therefore called the CF2N filters, is introduced in Section 5, and its performance is compared with TFL optimal filters obtained by a CR of degree 2. Design examples and a computational complexity comparison is presented in Section 6.

\section{Background}

\subsection{OFDM and FMT modulations}

The discrete-time version of a MC exponentially modulated signal may be written as

$$
s[k]=\sum_{n} \sum_{m=0}^{M-1} c_{m, n} p[k-n N] e^{j 2 \pi \frac{m}{M}}
$$

with $c_{m, n}$ the complex random information symbols to be transmitted on the $m$-th subcarrier of the $n$-th MC symbol and $p[k]$ the prototype filter of length $L$.

As all these types of MC systems involve a $e^{j 2 \pi \frac{m}{M}}$ kernel, they can take advantage of an Inverse Fast Fourier Transform (IFFT) realization.

The CP-OFDM and the Zero Padded OFDM (ZP-OFDM) both use a rectangular window of length $L=N$ so that each MC symbol can be process independently. So for CP-OFDM each symbol is associated with a prototype filter that can be expressed as

$$
p_{c p}[n]= \begin{cases}1, & 0 \leq n \leq N-1 \\ 0, & \text { otherwise }\end{cases}
$$

Note in this case that only the $M$-IFFT outputs contain the useful information. Indeed for each symbol the $N-M$ first ones are a copy of the tail samples of the IFFT output. Otherwise said the CP is of length $N-M$.

For ZP-OFDM, the prototype filter reads as

$$
p_{z p}[n]= \begin{cases}1, & 0 \leq n \leq M-1 \\ 0, & M \leq n \leq N-1\end{cases}
$$

So that both systems have a SE equal to $\frac{M}{N}$, which is also the case for FMT systems.

The goal with FMT systems, as proposed in [5], is to reduce the overlapping in frequency between consecutive subcarriers. To do so the oversampling factor $\frac{N}{M}$, inherent to this system when $N>M$, was used to increase, with respect to CP and ZP-OFDM, the frequency subspacing in a similar ratio without extending the time symbol duration, letting it be of $M$ samples. Secondly, for conventional FMT, again to limit intercarrier interference, the prototype filter length is, generally, relatively high $L \gg N$.

But, as illustrated in $[6,7,13]$, another option, more compatible with the numerous CP and ZP-OFDM communication standards, is to extend the time duration by a factor $\frac{N}{M}$, with $N>M$, and to keep the same subcarrier spacing as CP and ZP-OFDM. By the way, it is worth noting that the discrete-time expression (1) remains unchanged for both situations and also similar to the one used for defining oversampled OFDM systems in [14]. 


\subsection{PR conditions for FMT systems}

The PR property means that for the back-to-back FMT modulation system, up to a processing delay, the demodulated symbols $\hat{c}_{m, n}$ are proportional to the transmitted symbols, i.e. $\hat{c}_{m, n}=\gamma c_{m, n}$, with $\gamma>0$. As in $[14,5]$, we assume a matched filtering at the receiver side. Then, for general values of $M$ and $N$ with $M<N$, the PR relation for a prototype filter $P(z)=\sum_{n=0}^{L-1} p[n] z^{-n}$ of length $L$ may be written (cf. [9, equation (2)])

$$
\sum_{\nu} p[k+\nu M] p[k+\nu M+s N]=\delta_{s}, 0 \leq k \leq M-1, s \geq 0,
$$

where $\delta_{s}$ is the Kronecker symbol equal to 1 if $s=0$ and equal to 0 elsewhere. In (4), by convention, $p[n]=0$ if $n<0$ or $n \geq L$.

For MC systems of length $L=N$, the PR relations (4) become

$$
\begin{aligned}
& p[k]^{2}+p[k+M]^{2}=1,0 \leq k \leq N-M-1, \\
& p[k]^{2}=1, N-M \leq k \leq M-1 .
\end{aligned}
$$

As noted in [3], the ZP-OFDM system satisfies this condition as long as the receiver side also uses a $p_{z p}$ prototype filter. As mentioned in [6], the conventional CP-OFDM system does not satisfy the PR condition but biorthogonality conditions with a $M$-length rectangular window at the receiver side.

However, ZP or CP-OFDM prototype filters are not satisfactory with regard to their bad frequency behavior, and our goal, as in [3], is to find short PR prototype filters being optimal with respect to the TFL criterion.

\subsection{Time-frequency localization criterion}

Following Doroslovački [15], the time-frequency localization $\xi$ of a length $L$ filter $P(z)=$ $\sum_{n=0}^{L-1} p[n] z^{-n}$ is defined by the following formulas:

$$
\begin{aligned}
T & =\frac{\sum_{n}\left(n-\frac{1}{2}\right)(p[n]+p[n-1])^{2}}{\sum_{n}(p[n]+p[n-1])^{2}}, \\
\|P\| & =\left(\sum_{n} p[n]^{2}\right)^{\frac{1}{2}}, \\
m_{2} & =\frac{1}{4\|P\|^{2}} \sum_{n}\left(n-\frac{1}{2}-T\right)^{2}(p[n]+p[n-1])^{2}, \\
M_{2} & =\frac{1}{\|P\|^{2}} \sum_{n}(p[n]-p[n-1])^{2}, \\
\xi & =\frac{1}{\sqrt{4 m_{2} M_{2}}} .
\end{aligned}
$$

with $m_{2}$ and $M_{2}$ the second order moments in time and frequency, respectively. For this discrete time measure, as also the case for the counterpart measure used for continuous time signals, we have $0 \leq \xi \leq 1$. 
In [3], we have proposed a prototype filter of length $L=N$ that not only satisfies (5) and (6) but is also nearly optimal with respect to the TFL criterion. As it is derived from a continuous time RRC function which is the dual of the RRC function often used in FMT systems [5], we named it dRRC. It is given by

$$
p_{\mathrm{dRRC}}= \begin{cases}\sin \left(\frac{(n+1) \pi}{2(N-M+1)}\right), & 0 \leq n \leq N-M-1 \\ 1, & N-M \leq n \leq M-1 \\ \sin \left(\frac{(N-n) \pi}{2(N-M+1)}\right), & M \leq n \leq N-1\end{cases}
$$

Note that with the resulting $N$-length FMT system, similarly to ZP and CP-OFDM, there is no overlapping in time between consecutive MC symbols and therefore no dependency. However, according to the application at hand, the TFL gain brought by the dRRC may be judged insufficient. So, in the following sections, we slightly relax the length constraint with an overlapping factor equal to 2 .

\section{$3 \quad$ PR conditions and angular representation}

In [3], prototype filters with PR property for parameters $M, M$ with $2 \leq M<N<2 M$ and length $L=N$ were studied. When $N=M+1$, it can be shown that there do not exist filters with a length $L$ such that $N<L<2 N$ satisfying the PR relations. That is not true when $N \neq M+1$ as proved by the following example.

Example 3.1. For $M=3, N=5$ and $L=7$, if $P(z)=\sum_{n=0}^{6} p[n] z^{-n}$ satisfies the $P R$ relations with $p[0] \neq 0$ and $p[6] \neq 0$, there exist two angles $\alpha_{0}, \alpha_{1}$ and $\varepsilon_{1}, \varepsilon_{2}= \pm 1$ such that

$$
P(z)=\sin \alpha_{0}+\varepsilon_{0} z^{-2}+\cos \alpha_{0} \sin \alpha_{1} z^{-3}+\varepsilon_{1} z^{-4}+\cos \alpha_{0} \cos \alpha_{1} z^{-6} .
$$

However, in all the following of this paper, we consider prototype filters with PR property for parameters $M$ and $N$ with $2 \leq M<N<2 M$ and length $L=2 N$.

First, remark that non trivial relations in (4) are obtained for $s=0$ or $s=1$ because for $s \geq 2, k+\nu M+s N \geq s N \geq 2 N$ and thus $p[k+\nu M+s N]=0$ for any value of $k, 0 \leq k \leq M-1$ and $\nu \geq 0$.

The PR relations obtained for $s=1$ are called the orthogonality PR relations. Such a relation with three terms, for $0 \leq k \leq M-1$, can be written

$$
p[k] p[k+N]+p[k+M] p[k+M+N]+p[k+2 M] p[k+2 M+N]=0
$$

However, because $N<2 M$, we have $k+2 M+N>2 N$ and thus $p[k+2 M+N]=0$. So $\mathrm{PR}$ orthogonality relations have at most two terms

$$
p[k] p[k+N]+p[k+M] p[k+M+N]=0 .
$$

In (13), the second term of the left member is null if and only if $k+M+N \geq 2 N$, i.e. $N-M \leq k \leq M-1$. This proves that the $M \mathrm{PR}$ orthogonality relations may be written

$$
\begin{aligned}
& p[k] p[k+N]+p[k+M] p[k+M+N]=0,0 \leq k \leq N-M-1, \\
& p[k] p[k+N]=0, N-M \leq k \leq M-1 .
\end{aligned}
$$


In order to minimize the function $M_{2}$ for the filters, we choose the $2 M-N$ null values implied by equations (15) in contiguous places, and more precisely, $p[k+N]=0, N-M \leq$ $k \leq M-1$, i.e. $p[k]=0,2 N-M \leq k \leq M+N-1$. The other choice, $p[k]=0, N-M \leq$ $k \leq M-1$, leads to their symmetrical filters.

We consider now the PR relations obtained for $s=0$, called the normalization $P R$ relations. For any $k, 0 \leq k \leq M-1$, we have $k+4 M>2 N$ because $N<2 M$. So the number of terms in a normalization relation is at most 4 :

$$
p[k]^{2}+p[k+M]^{2}+p[k+2 M]^{2}+p[k+3 M]^{2}=1,0 \leq k \leq M-1 .
$$

However the fourth term of the left member is always null if $2 N \leq 3 M$, i.e. $\rho=\frac{N-M}{M} \leq \frac{1}{2}$.

Two cases must be considered, and we can remark that the limit case between them, $2 N=3 M$ can be considered as well by the first or the second case description.

\subsection{Case 1: $2 N \leq 3 M$}

As shown in Figure 1, normalization PR relations belong to three subcases.

- Case 1.a. The $N-M$ relations

$$
p[k]^{2}+p[k+M]^{2}=1,0 \leq k \leq N-M-1 .
$$

- Case 1.b. The $N-M$ relations

$$
p[k]^{2}+p[k+M]^{2}+p[k+2 M]^{2}=1, N-M \leq k \leq 2(N-M)-1 .
$$

- Case 1.c. The $3 M-2 N$ relations

$$
p[k]^{2}=1,2(N-M) \leq k \leq M-1 .
$$

We then choose, from equations $(19), p \mid k]=1,2(N-M) \leq k \leq M-1$, in order to minimize the $M_{2}$ function of the filter. Rewriting equations (18) as

$$
p[k+N-M]^{2}+p[k+N]^{2}+p[k+N+M]^{2}=1,0 \leq k \leq N-M-1,
$$

one remarks that equations (17), (20) and (14) may be written as a set of $N-M$ similar systems $\mathcal{S}_{k}, 0 \leq k \leq N-M-1$, where

$$
\mathcal{S}_{k}=\left\{\begin{array}{l}
p[k]^{2}+p[k+M]^{2}=1 \\
p[k+N-M]^{2}+p[k+N]^{2}+p[k+N+M]^{2}=1, \\
p[k] p[k+N]+p[k+M] p[k+M+N]=0
\end{array}\right.
$$

and where the set of variables of $\mathcal{S}_{k}$ is $s_{k}=\{p[k], p[k+N-M], p[k+M], p[k+N], p[k+M+N]\}$. The sets $s_{k}, 0 \leq k \leq N-M-1$ are disjoint and thus the $\mathcal{S}_{k}$ systems are independent.

From equation $p[k]^{2}+p[k+M]^{2}=1$ it follows that there exists an angle $\alpha_{k}$ such that $p[k]=\sin \alpha_{k}, p[k+M]=\cos \alpha_{k}$. Thus equation $p[k] p[k+N]+p[k+M] p[k+M+N]=0$ 


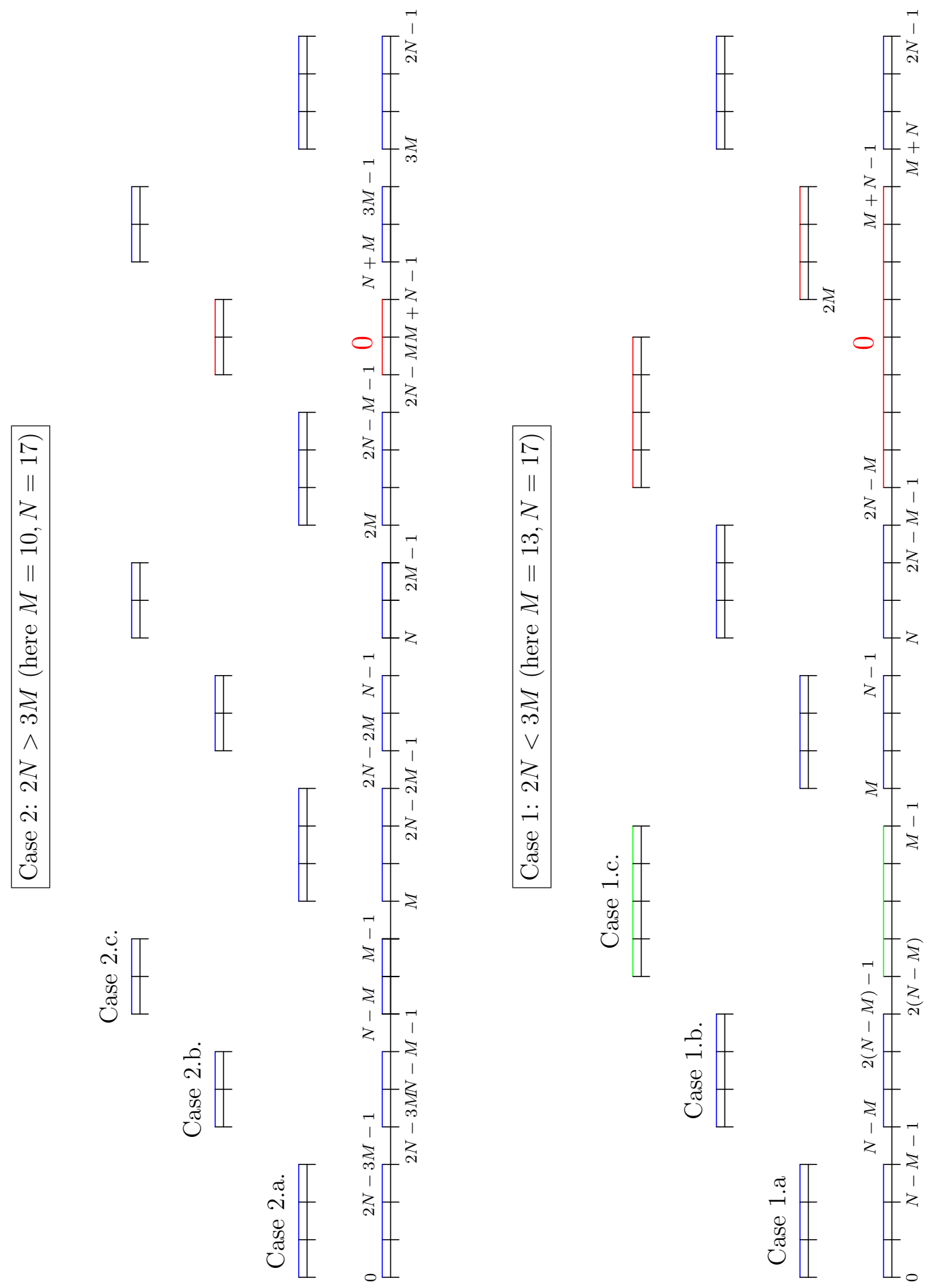

Figure 1: A schematic representation of normalization PR relations for PR FMT filters with $L=2 N$. 
may be written $p[k+N] \sin \alpha_{k}+p[k+M+N] \cos \alpha_{k}=0$ and there exists a number $b_{k}$ such that $p[k+N]=b_{k} \cos \alpha_{k}$ and $p[k+M+N]=-b_{k} \sin \alpha_{k}$. Reporting in equation $p[k+N-M]^{2}+p[k+N]^{2}+p[k+N+M]^{2}=1$ gives $p[k+M-N]^{2}+b_{k}^{2}=1$. Therefore there exists an angle $\beta_{k}$ such that $p[k+M-N]=\sin \beta_{k}$ and $b_{k}=\cos \beta_{k}$.

We conclude that there exist two angles $\alpha_{k}$ and $\beta_{k}$ such that the solution of $\mathcal{S}_{k}$ system may be written

$$
\begin{array}{ll}
p[k] & =\sin \alpha_{k}, \\
p[k+N-M] & =\sin \beta_{k}, \\
p[k+M] & =\cos \alpha_{k}, \\
p[k+N] & =\cos \alpha_{k} \cos \beta_{k}, \\
p[k+M+N] & =-\sin \alpha_{k} \cos \beta_{k} .
\end{array}
$$

\subsection{Case 2: $2 N \geq 3 M$}

As shown by the example $M=10, N=17$ in Figure 1 , the $M$ normalization PR equations belong to three subcases.

- Case 2.a. The $2 N-3 M$ equations

$$
p[k]^{2}+p[k+M]^{2}+p[k+2 M]^{2}+p[k+3 M]^{2}=1,0 \leq k \leq 2 N-3 M-1 .
$$

- Case 2.b. The $2 M-N$ equations

$$
p[k]^{2}+p[k+M]^{2}=1,2 N-3 M \leq k \leq N-M-1 .
$$

- Case 2.c. The $2 M-N$ equations

$$
p[k]^{2}+p[k+M]^{2}+p[k+2 M]^{2}=1, N-M \leq k \leq M-1 .
$$

In contrast with previous Case 1 , it is not possible to split the PR equations in similar independent systems.

Using relation (13), for $0 \leq k \leq N-M-1$, let us define an angle $\alpha_{k}$ and two constants $b_{k}$ and $c_{k}$ such that

$$
\begin{array}{lll}
p[k] & = & b_{k} \sin \alpha_{k}, \\
p[k+M] & = & b_{k} \cos \alpha_{k}, \\
p[k+N] & = & c_{k} \cos \alpha_{k}, \\
p[k+M+N] & = & -c_{k} \sin \alpha_{k} .
\end{array}
$$

Using (26) in (24) gives $b_{k}^{2}=1$ for $2 N-3 M \leq k \leq N-M-1$, thus we may choose $b_{k}=1$ for $2 N-3 M \leq k \leq N-M-1$ and

$$
p[k]=\sin \alpha_{k}, p[k+M]=\cos \alpha_{k}, 2 N-3 M \leq k \leq N-M-1 .
$$

A change of indices $k=k^{\prime}+N-M, k^{\prime} \rightarrow k$, allows to rewrite equations (25) as

$$
p[k+N-M]^{2}+p[k+N]^{2}+p[k+M+N]^{2}=1,0 \leq k \leq 2 M-N-1,
$$


and using (26) $p[k+N]=c_{k} \cos \alpha_{k}, p[k+M+N]=-c_{k} \sin \alpha_{k}$, we get

$$
p[k+N-M]^{2}+c_{k}^{2}=1,0 \leq k \leq 2 M-N-1 .
$$

Thus there exist $2 M-2 N$ angles $\beta_{k}, 0 \leq k \leq 2 M-N-1$, such that $p[k+N-M]=\sin \beta_{k}$ and $c_{k}=\cos \beta_{k}$, and it follows that, for $0 \leq k \leq 2 M-N-1$,

$$
\begin{array}{ll}
p[k+N-M] & =\sin \beta_{k}, \\
p[k+N] & =\cos \alpha_{k} \cos \beta_{k}, \\
p[k+M+N] & =-\sin \alpha_{k} \cdot \cos \beta_{k} .
\end{array}
$$

Using (26), we have $p[k+2 M]=p[k+2 M-N+N]=c_{k+2 M-N} \cos \alpha_{k+2 M-N}$ and $p[k+3 M]=p[k+2 M-N+N+M]=-c_{k+2 M-N} \sin \alpha_{k+2 M-N}$, and thus, from equations $(23)$,

$$
b_{k}^{2}+c_{k+2 M-N}^{2}=1,0 \leq k \leq 2 N-3 M-1,
$$

or

$$
b_{k-2 M+N}^{2}+c_{k}^{2}=1,2 M-N \leq k \leq N-M-1,
$$

Therefore there exist $2 N-3 M$ angles, $\beta_{k}, 2 M-N \leq k \leq N-M-1$, such that $b_{k-2 M+N}=\sin \beta_{k}$ and $c_{k}=\cos \beta_{k}$, and we get, for $2 M-N \leq k \leq N-M-1$,

$$
\begin{array}{ll}
p[k-2 M+N] & =\sin \alpha_{k-2 M+N} \sin \beta_{k}, \\
p[k-M+N] & =\cos \alpha_{k-2 M+N} \sin \beta_{k}, \\
p[k+N] & =\cos \alpha_{k} \cos \beta_{k}, \\
p[k+M+N] & =-\sin \alpha_{k} \cos \beta_{k} .
\end{array}
$$

In conclusion, in the case $2 N \geq 3 M$, a filter $P(z)=\sum_{k=0}^{2 N-1} p[n] z^{-n}$ with the PR property is completely determined by $2(N-M)$ angles $\alpha_{k}, \beta_{k}, 0 \leq k \leq N-M+1$ with equations $p[k]=0,2 N-M \leq k \leq M+N-1,(27),(30)$ and (33).

\subsection{A general algorithm}

In both cases $2 N \leq 3 M$ and $2 N \geq 3 M$, both methods to construct a PR filter $P(z)=$ $\sum_{k=0}^{2 N-1} p[n] z^{-n}$ of length $L=2 N$ with PR property for parameters $M$ and $N$ with $2 \leq$ $M<N \leq 2 M-1$ use $2(N-M)$ angular parameters $\alpha_{k}, \beta_{k}, 0 \leq k \leq N-M-1$. It is straightforward to check that in the case of an even value of $M$ and if $N=3 M / 2$, the two constructions give the same result.

It is now possible to give a general algorithm to compute coefficients $p[k], 0 \leq k \leq 2 N-1$. 


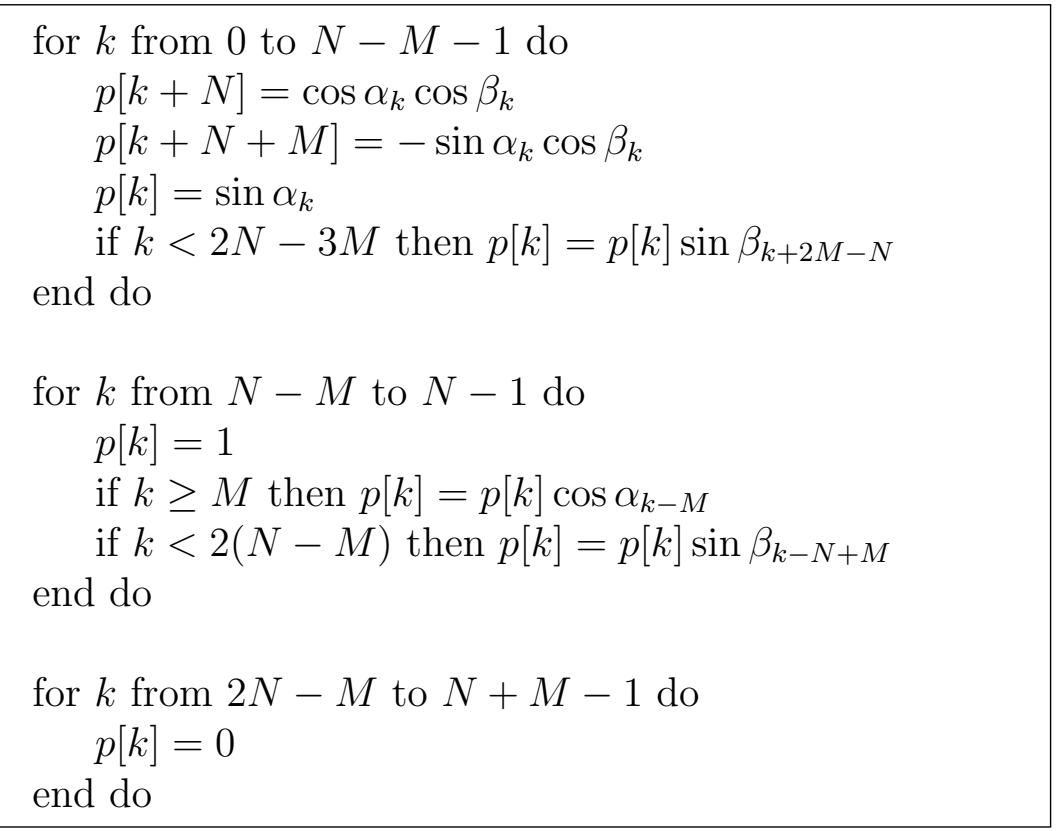

Remark.- Denoting by $P\left(\alpha_{0}, \alpha_{1}, \ldots, \alpha_{N-M-1} ; \beta_{0}, \beta_{1}, \ldots, \beta_{N-M-1} ; z\right)$ the PR filter with length $L=2 N$ built by the above algorithm and by $P\left(\alpha_{0}, \alpha_{1}, \ldots, \alpha_{N-M-1} ; z\right)$ the PR filter with length $L=N$ studied in [3], it is straightforward to observe that

$$
\begin{aligned}
P\left(\alpha_{0}, \alpha_{1}, \ldots, \alpha_{N-M-1} ; \frac{\pi}{2}, \frac{\pi}{2}, \ldots, \frac{\pi}{2} ; z\right) & =P\left(\alpha_{0}, \alpha_{1}, \ldots, \alpha_{N-M-1} ; z\right) \\
P\left(0,0, \ldots, 0 ; \beta_{0}, \beta_{1}, \ldots, \beta_{N-M-1} ; z\right) & =z^{-(N-M)} P\left(\beta_{0}, \beta_{1}, \ldots, \beta_{N-M-1} ; z\right) .
\end{aligned}
$$

\section{TFL optimization using a compact representation}

\subsection{TFL optimization using angles}

For given values of $M$ and $N$ with $2 \leq M<N<2 M$, the optimization of the TFL for a PR filter with length $L=2 N$ appears as an optimization problem with $2(N-M)$ angular parameters $\alpha_{k}, \beta_{k}, 0 \leq k \leq N-M-1$. When $2(N-M)$ is small enough, a program like CFSQP ([16]) is achieving satisfactory results. As a matter of example, Figure 2 shows the values of angles $\alpha_{k}, \beta_{k}$ as functions of $k, 0 \leq k \leq N-M-1$ that provide the optimal TFL for $M=40, N=58$ (a Case 1 situation) or $M=40, N=68$ (a Case 2 situation).

By placing the graphs of the $\alpha_{k}$ and the graphs of the $\beta_{k}$ beside each other, we get the graphs given in Figure 3. More precisely, defining $\gamma_{k}=\alpha_{k}$ for $0 \leq k \leq N-M-1$ and $\gamma_{k}=\beta_{k-N+M}, N-M \leq k \leq 2(N-M)-1$, Figure 3 shows the sets of points $\left(k, \gamma_{k}\right)$ for our two examples.

In both Figures 2 and 3, points seem to be located on regular curves, which is the basis of the compact representations developed in the next sub-section. We must emphasize on the fact that the same behavior occurs with the choice of other values of $M$ and $N$. 

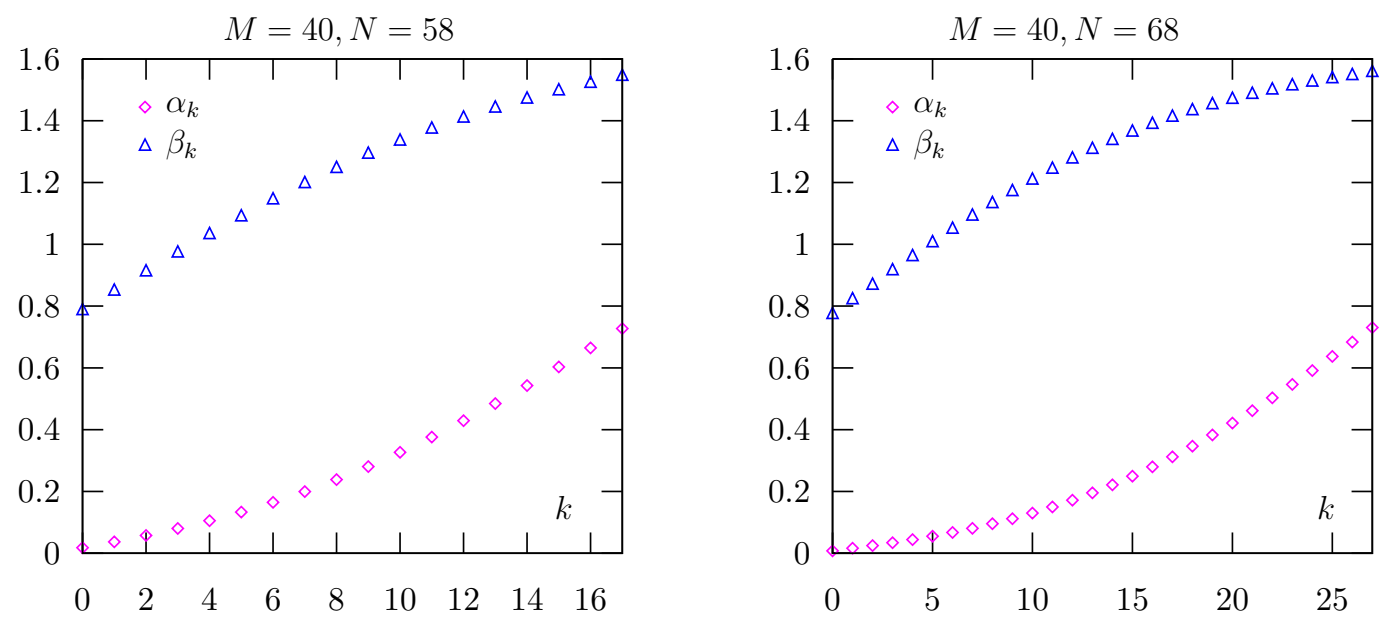

Figure 2: Angles $\alpha_{k}, \beta_{k}, 0 \leq k \leq N-M-1$ for optimal TFL filters with $M=40, N=58$ or $M=40, N=68$.
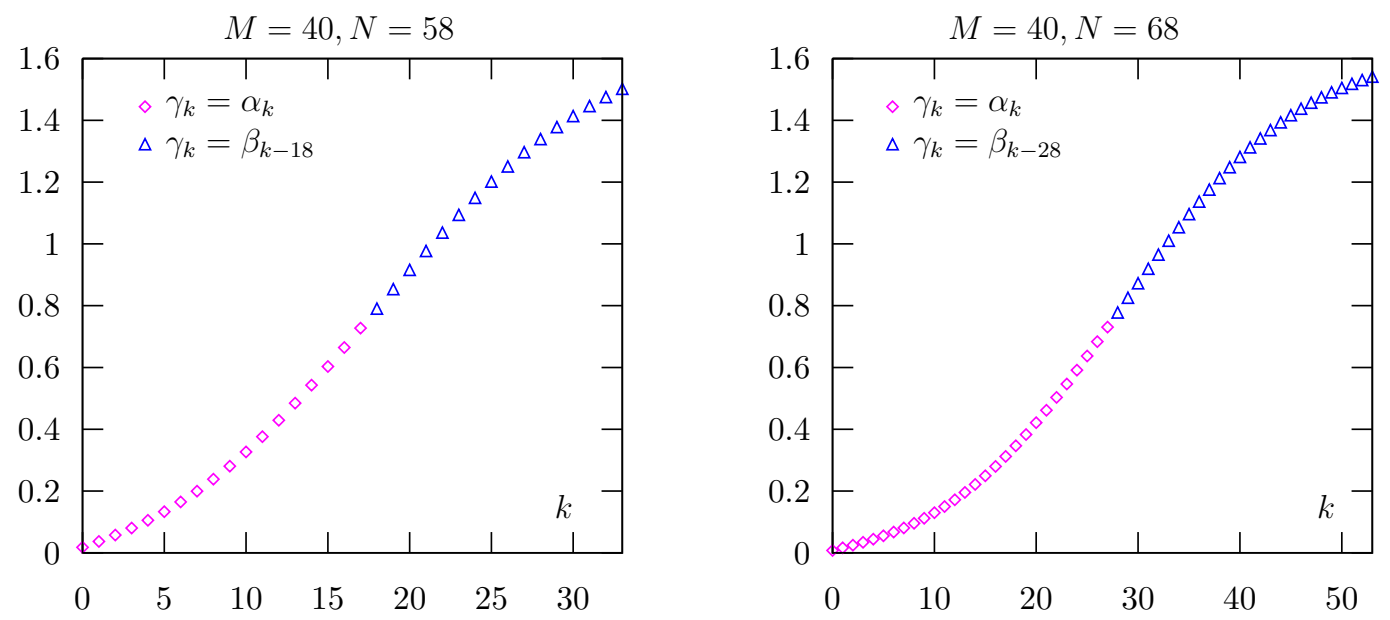

Figure 3: Angles $\gamma_{k}, 0 \leq k \leq 2(N-M)-1$ for optimal TFL filters with $M=40, N=58$ or $M=40, N=68$. 


\subsection{Angular compact representation}

The previous analysis clearly shows that a CR, such as presented in [17], could be used to solve the TFL optimization problem. To do so, we first need an abscissa function $k \in$ $\{0,1, \ldots, N-M-1\} \Rightarrow x(k) \in(0,1)$, and then angular functions $\alpha(x)$ and $\beta(x)$ defined on $(0,1)$ to define the angles $\alpha_{k}$ and $\beta_{k}, 0 \leq k \leq N-M-1$ by

$$
\alpha_{k}=\frac{\pi}{2} \alpha(x(k)), \beta_{k}=\frac{\pi}{2} \beta(x(k))
$$

Functions $\alpha(x)$ and $\beta(x)$ are supposed to only depend on a few coefficients, for example, for a given integer $d$, the coefficients of a polynomial of degree $d-1$ :

$$
\alpha(x)=\sum_{i=0}^{d-1} a_{i} x^{i}, \beta(x)=\sum_{i=0}^{d-1} b_{i} x^{i} .
$$

The number $d$ is called the CR degree. Then, the TFL optimization problem involves $d$ pairs of $\left(a_{i}, b_{i}\right)$ coefficients instead of $2(N-M)$ angles.

We may use the abscissa functions

$$
x_{1}(k)=\frac{2 k+1}{2(N-M)}, 0 \leq k \leq N-M-1, \quad(\mathrm{CR} 1)
$$

as in [17] or

$$
x_{2}(k)=\frac{k+1}{N-M+1}, 0 \leq k \leq N-M-1,
$$

as in [3]. CR1 and CR2 give absolutely the same results for an optimization of the TFL criterion, for given values of $M, N$ and $d$, because $x_{2}(k)$ is a linear function of $x_{1}(k)$

$$
x_{2}(k)=\frac{1}{2(N-M+1)}+\frac{N-M}{N-M+1} x_{1}(k), 0 \leq k \leq N-M-1 .
$$

However, for fixed functions $\alpha(x)$ and $\beta(x)$, the two abscissa functions do not provide the same filter, as we will see later on.

Figure 3 suggests choosing a single function $\gamma(x)=\sum_{i=0}^{d-1} c_{i} x^{i}$ and to define the angles $\alpha_{k}$ and $\beta_{k}, 0 \leq k \leq N-M-1$ by

$$
\begin{aligned}
\alpha_{k} & =\frac{\pi}{2} \gamma\left(x_{1}(k)\right), 0 \leq k \leq N-M-1, \\
\beta_{k} & =\frac{\pi}{2} \gamma\left(x_{1}(k+N-M)\right), 0 \leq k \leq N-M-1,
\end{aligned}
$$

where the abscissa $x_{1}(k)$ is defined on $\{0,1, \ldots, 2(N-M)-1\}$ by $x_{1}(k)=\frac{2 k+1}{4(N-M)}(\mathrm{CR} 3)$. A similar definition uses the abscissa function $x_{2}(k)=\frac{k+1}{2(N-M)+1}$ defined on the same set (CR 4). For CR3 and CR4, the TFL optimization is carried out over the $c_{i}$ coefficients, i.e. using $d$ variables. 
Figure 3 also suggests that such a function $\gamma(x)$ verifies a symmetry property, $\gamma(1-x)=$ $1-\gamma(x), x \in(0,1)$ and then an adapted CR would be

$$
\gamma(x)=\frac{1}{2}+\sum_{i=0}^{d-1} c_{i}\left(x-\frac{1}{2}\right)^{2 i+1}
$$

choosing $x_{1}(k)$ as abscissa function (CR 5) or $x_{2}(k)$ (CR 6) as done in [3]). As for CR3, CR4 we optimize over $d$ variables but then using a polynomial of degree $2 d-1$ instead of $d-1$.

To compare these six compact representations, we present in Table 1 what are the optimum TFL obtained by using each of the four first CR with $d=2$ and $d=5$ for the examples in Figures 2 and 3. For CR 5 and CR $6, d=3$ also corresponds to a symmetrical polynomial of degree 5. So, for all these cases, the number of $\mathrm{CR}$ variables goes from 1 (CR5, CR6 for $d=1$ ) up to 10 (e.g. CR1, CR2 for $d=5$ ) while with the angular parameters representation we must deal with 18 or 28 variables. More important is that the optimization using the $2(N-M)$ angular parameters, or directly the $2 N$ filter coefficients, becomes quickly untractable if one targets the design of FMT systems with hundreds or thousands of coefficients.

Although it is obvious, from Table 1, that the symmetrical representations give almost optimal results, in the rest of the paper, we will use the CRs providing the best results, i.e. CR 1 or CR 2,

\begin{tabular}{|c|c|c||c|}
\hline & $d$ & $M=40, N=58$ & $M=40, N=68$ \\
\hline \hline \multicolumn{2}{|c|}{ With angles } & 0.8553027 & 0.9371121 \\
\hline \hline CR 1 & 2 & 0.8021287 & 0.8625798 \\
\hline CR 2 & 2 & 0.8021287 & 0.8625798 \\
\hline CR 3 & 2 & 0.7970992 & 0.8253963 \\
\hline CR 4 & 2 & 0.7970992 & 0.8533307 \\
\hline CR 5 & 1 & 0.7970805 & 0.8253712 \\
\hline CR 6 & 1 & 0.7970805 & 0.8253712 \\
\hline \hline CR 1 & 5 & 0.8553006 & 0.9371045 \\
\hline CR 2 & 5 & 0.8553007 & 0.9371045 \\
\hline CR 3 & 5 & 0.8533307 & 0.9296053 \\
\hline CR 4 & 5 & 0.8533307 & 0.9296053 \\
\hline CR 5 & 3 & 0.8551227 & 0.9352910 \\
\hline CR 6 & 3 & 0.8550895 & 0.9347470 \\
\hline
\end{tabular}

Table 1: Comparing six CR for TFL optimization with $M=40, N=58$ and $M=40, N=$ 68. 


\section{CF2N filters and TFL optimization}

\subsection{CF2N filters and first examples}

A simple choice of function $\alpha(x)$ and $\beta(x)$ for CR 1 and CR 2, linear in variable $x$ and not depending on $M$ and $N$, with $M<N<2 M$, gives a fairly good approximation of the best prototype filter of length $2 N$ for the TFL criterion for small values of $\rho=\frac{N-M}{M}$ :

$$
\alpha(x)=\frac{x}{2}, \quad \beta(x)=\frac{1}{2}+\frac{x}{2} .
$$

Such filters that, similarly to the dRRC filters of length $L=N$ presented in [3], can be simply expressed by a closed form expression, will be called CF2N filters. We consider also the linear function $\gamma(x)=x$ for CR 3 and CR 4, or with the here equivalent symmetric compact representations. Table 2 gives the values of the TFL and it appears that CR1 or CR3 provides the best TFL. Thus in the following, we choose the CR 1 to define the CF2N filter.

\begin{tabular}{|l|c||c|}
\hline & $M=40, N=58$ & $M=40, N=68$ \\
\hline \hline CR 1 & 0.7965006 & 0.8239771 \\
\hline CR 2 & 0.7901057 & 0.8198295 \\
\hline CR 3 & 0.7965006 & 0.8239771 \\
\hline CR 4 & 0.7863118 & 0.8153444 \\
\hline
\end{tabular}

Table 2: TFL values of CF2N filters for $M=40, N=58$ or $M=40, N=68$ with CR 1 to CR 4.

Table 3 gives the TFL values of the CF2N filter together with the optimized TFL for different values of $(M, N)$ and different CR1 (or, equivalently, CR2) degrees, i.e. with two angle functions and the abscissa functions $x_{1}(k)(\mathrm{CR} 1)$, or $x_{2}(k)$ (CR 2) in an equivalent way. The $(M, N)$ parameters values for the LTE1, LTE2, P1901 and DVB-T2 standards

\begin{tabular}{|c|c|c|c|c|c|c|}
\hline$(M, N)$ & $(40,58)$ & $(40,68)$ & LTE1 & LTE2 & P1901 & DVB-T2 \\
\hline CF2N & 0.7965006 & 0.8239771 & 0.3967366 & 0.3903139 & 0.6827997 & 0.2625591 \\
$d=2$ & 0.8021287 & 0.8625798 & 0.3989285 & 0.3905006 & 0.6829892 & 0.2625959 \\
$d=3$ & 0.8548504 & 0.9360846 & 0.4016335 & 0.3940837 & 0.7057080 & 0.2643995 \\
$d=4$ & 0.8549569 & 0.9362178 & 0.4016515 & 0.3941222 & 0.7058168 & 0.2644241 \\
$d=5$ & 0.8553007 & 0.9371046 & 0.4016572 & 0.3941396 & 0.7059988 & 0.2644313 \\
$d=6$ & 0.8553022 & 0.9371116 & 0.4016573 & 0.3941399 & 0.7060006 & 0.2644313 \\
$d=7$ & 0.8553026 & 0.9371118 & 0.4016573 & 0.3941400 & 0.7060008 & 0.2644313 \\
\hline
\end{tabular}

Table 3: Prototype filters optimized for the TFL criterion with different values of $(M, N)$, $L=2 N$ and increasing degrees for CR 1 (or, equivalently, CR 2). 
are equal to $(128,137),(2048,2192),(8192,10296)$ and $(32768,33792)$, respectively. We may remark that the optimal TFL is almost obtained with $d=5$.

\subsection{The particular case: $N=M+1$}

Unlike the case of the dRRC filter in [3], we have not found an explicit formula for the TFL $\xi_{\mathrm{CF} 2 \mathrm{~N}}(M)$ of the CF2N filter when $N=M+1$. However, a least square method leads to the approximation

$$
\xi_{\mathrm{CF} 2 \mathrm{~N}}(M) \simeq \frac{M}{\sqrt{\sum_{i=0}^{3} a_{i} M^{i}}}
$$

where

$$
\begin{array}{ll}
a_{0}=-0.2874744067, & a_{1}=1.5103273480 \\
a_{2}=-0.1418377584, & a_{3}=0.3146978688
\end{array}
$$

In particular, when $M$ tends to infinity,

$$
\xi_{\mathrm{CF} 2 \mathrm{~N}}(M) \sim \frac{A}{\sqrt{M}} \text { with } A=1.782596704 .
$$

In the same way, we get a similar approximation for $\xi_{1}(M)$, the optimized TFL for any compact representation (here $d=1$ is sufficient) :

$$
\xi_{1}(M) \simeq \frac{M}{\sqrt{\sum_{i=0}^{3} b_{i} M^{i}}},
$$

where

$$
\begin{array}{ll}
b_{0}=-1.7478302544, & b_{1}=1.6029244504, \\
b_{2}=-0.0475712071, & b_{3}=0.3005223238 .
\end{array}
$$

And in particular, when $M$ tends to infinity,

$$
\xi_{1}(M) \sim \frac{B}{\sqrt{M}} \text { with } B=1.82415455 .
$$

\subsection{TFL comparison: CF2N versus CR1 optimization with $d=2$}

For $M$ and $N$ with $2 \leq M<N<2 M$, we define now by $\xi_{\mathrm{CF} 2 \mathrm{~N}}(M, N)$ the TFL of the CF2N filter and by $\xi_{2}(M, N)$ the optimized TFL of a filter of length defined by a CR of degree 2. Then, we define the relative gain of the $d=2$ optimized filter w.r.t. the CF2N filter, $G_{\mathrm{CF} 2 \mathrm{~N}, 2}(M, N)$, by

$$
G_{\mathrm{CF} 2 \mathrm{~N}, 2}(M, N)=\frac{\xi_{2}(M, N)-\xi_{\mathrm{CF} 2 \mathrm{~N}}(M, N)}{\xi_{\mathrm{CF} 2 \mathrm{~N}}(M, N)} .
$$

The results of the previous paragraph lead to

$$
l_{0} \equiv \lim _{M \rightarrow \infty} G_{\mathrm{CF} 2 \mathrm{~N}, 2}(M, M+1)=\frac{B-A}{A}=0.023313 .
$$


It is preferable to represent the function $G_{\mathrm{CF} 2 \mathrm{~N}, 2}(M, N)$, for a given value of $M$, as a function of the relative redundancy $\rho=\frac{N-M}{M}$. In this way, Figure 4 represents the values of $G_{\mathrm{CF} 2 \mathrm{~N}, 2}(M, N)$ for $M=2^{k}, 5 \leq k \leq 11$ and $M+1 \leq N \leq 2 M-1$. Figure 5 is a zoom of Figure 4 for $0 \leq \rho \leq 0.6$, a region where the CF2N filter may well approximate the optimized filter for $d=2$. More precisely, it is shown for example in Figure 5 that $G_{\mathrm{CF} 2 \mathrm{~N}, 2}(M, N)<0.005$ when $M \geq 128$ if $\rho_{1}(0.005)=0.078125 \leq \rho \leq \rho_{2}(0.005)=0.484375$.

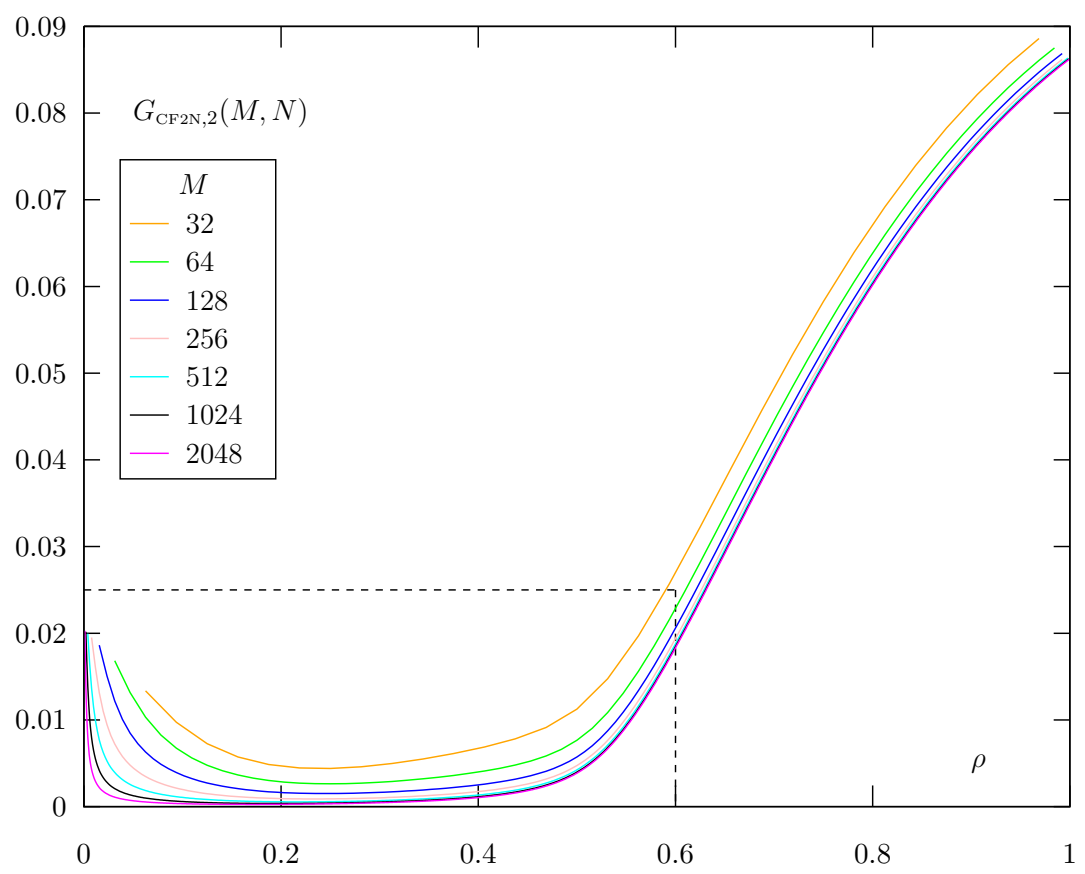

Figure 4: Relative gain $G_{\mathrm{CF} 2 \mathrm{~N}, 2}(M, N)$ for $M=2^{k}, 5 \leq k \leq 11$ and $M+1 \leq N \leq 2 M-1$.

\subsection{Limit of optimized TFL when $M$ tends to $\infty, \rho$ being constant}

For given $M_{0}$ and $N_{0}$ with $2 \leq M_{0}<N_{0}<2 M_{0}$, and $\Delta \geq 1$, we may compute the PR filter with optimized TFL for parameter $M=\Delta M_{0}, N=\Delta N_{0}$ and length $L=2 N$. When $\Delta$ tends to infinity, we observe that the optimal TFL, denoted by $\xi_{\text {opt }}(M, N)$ decreases but tends to a strictly positive limit and a first least squares approximation leads to an expression

$$
\xi_{\text {opt }}(M, N) \approx a_{0}(\rho)+\frac{a_{1}(\rho)}{M},
$$

where $\rho=\frac{N-M}{M}$. More generally, we may suppose that $\xi_{\text {opt }}(M, N)$ may be expressed as a series in $\frac{1}{M}$ and coefficients being functions of $\rho$

$$
\xi_{\text {opt }}(M, N)=a_{0}(\rho)+\frac{a_{1}(\rho)}{M}+\frac{a_{2}(\rho)}{M^{2}}+\frac{a_{3}(\rho)}{M^{3}}+\ldots
$$

Therefore, for a given fixed value of $\rho$ and using several values of $M$, the Richardson's extrapolation method [18] allows us to compute a precise approximation of the limit $a_{0}(\rho)$ when $M$ tends to infinity. 


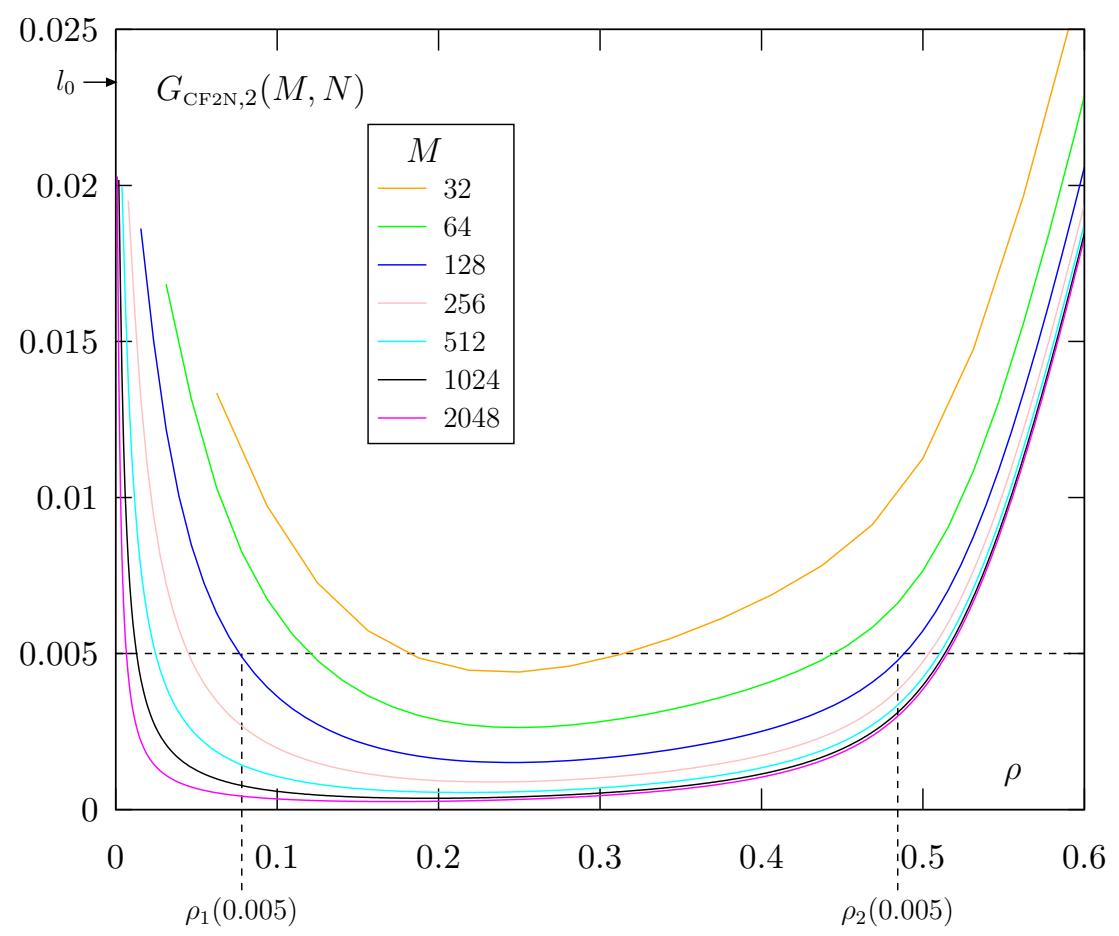

Figure 5: Relative gain $G_{\mathrm{CF} 2 \mathrm{~N}, 2}(M, N)$ for $M=2^{k}, 5 \leq k \leq 11$ and $M+1 \leq N \leq 2 M-1$ (zoom of Figure 4).

This method may be applied to the optimized TFL obtained with a CR of degree 5 or 2 , to the TFL of the CF2N filter, or of the optimized TFL for a PR filter of length $L=N$ as in [3]. We denote by $\xi_{\lim , 5}(\rho), \xi_{\lim , 2}(\rho), \xi_{\lim , \mathrm{CF} 2 \mathrm{~N}}(\rho), \xi_{\lim , L=N}(\rho)$ the corresponding limits when $M$ tends to infinity. Figure 6 shows the graphs of these functions of $\rho$. It is clear that the CF2N filter has a limited interest when $\rho>\frac{1}{2}$ and its TFL is even worse than the TFL of the $L=N$ optimized filter for large values of $\rho(\rho>0.72)$.

\section{Design examples and computational complexity}

\subsection{Time and frequency behavior}

In this paragraph we compare the best prototype filters we have obtained running our TFL optimization, for $L=N$, using the symmetric CR proposed in [3] for linear-phase prototype filter, and $L=2 N$ using CR1 with $d=5$.

Fig. 7 presents the PR prototype filter for a FMT system with 128 subcarriers and attaining the maximum possible oversampling ratio for the proposed method. One can first notice the clear advantage obtained in frequency by the $L=2 N$ prototype filter. Secondly, it has to be noted that the TFL measure, equal to 0.973869 , nearly attains the global optimal value $(\xi=1)$. In other words, this means that only a limited TFL gain can be expected with longer prototype filters.

Figs. 8 and 9 both correspond to LTE settings for which the relative redundancy is 


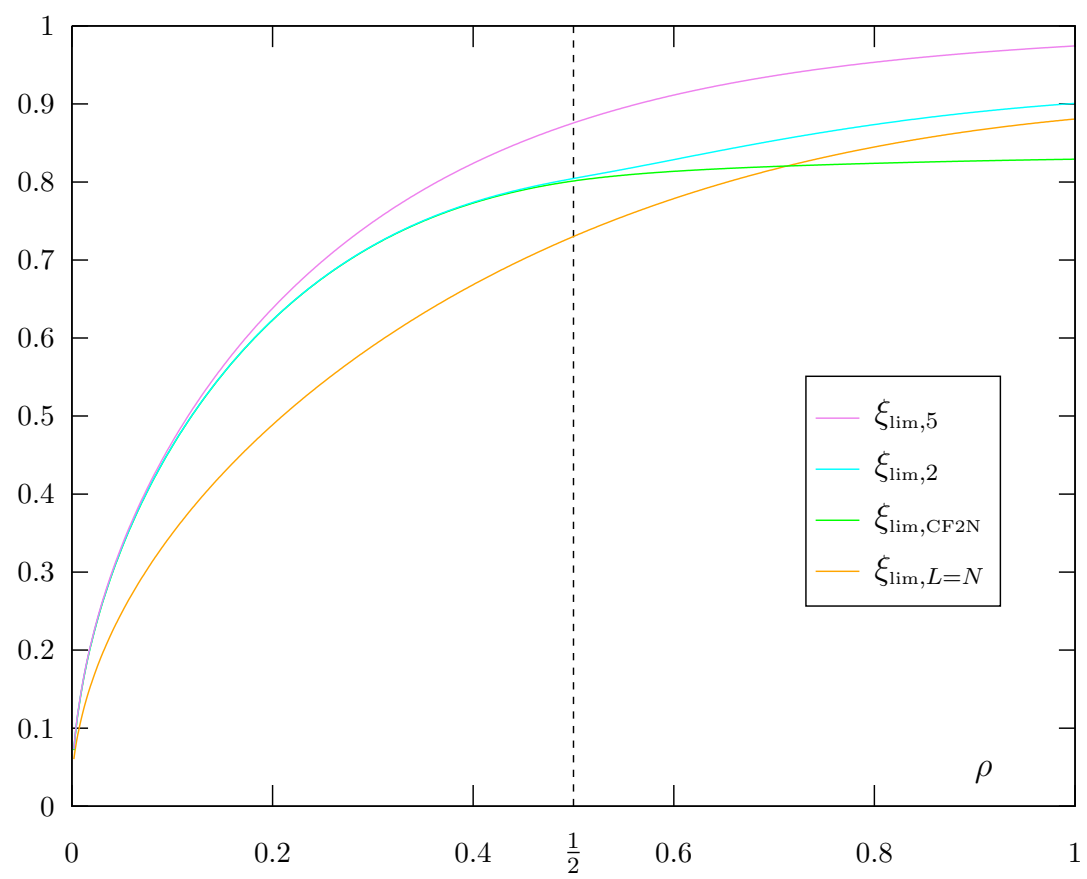

Figure 6: Limits of optimized TFL $\xi_{\lim , 5}(\rho), \xi_{\lim , 2}(\rho), \xi_{\lim , L=N}(\rho)$ and of $\xi_{\lim , \mathrm{CF} 2 \mathrm{~N}}(\rho)$ when $M \rightarrow \infty$.
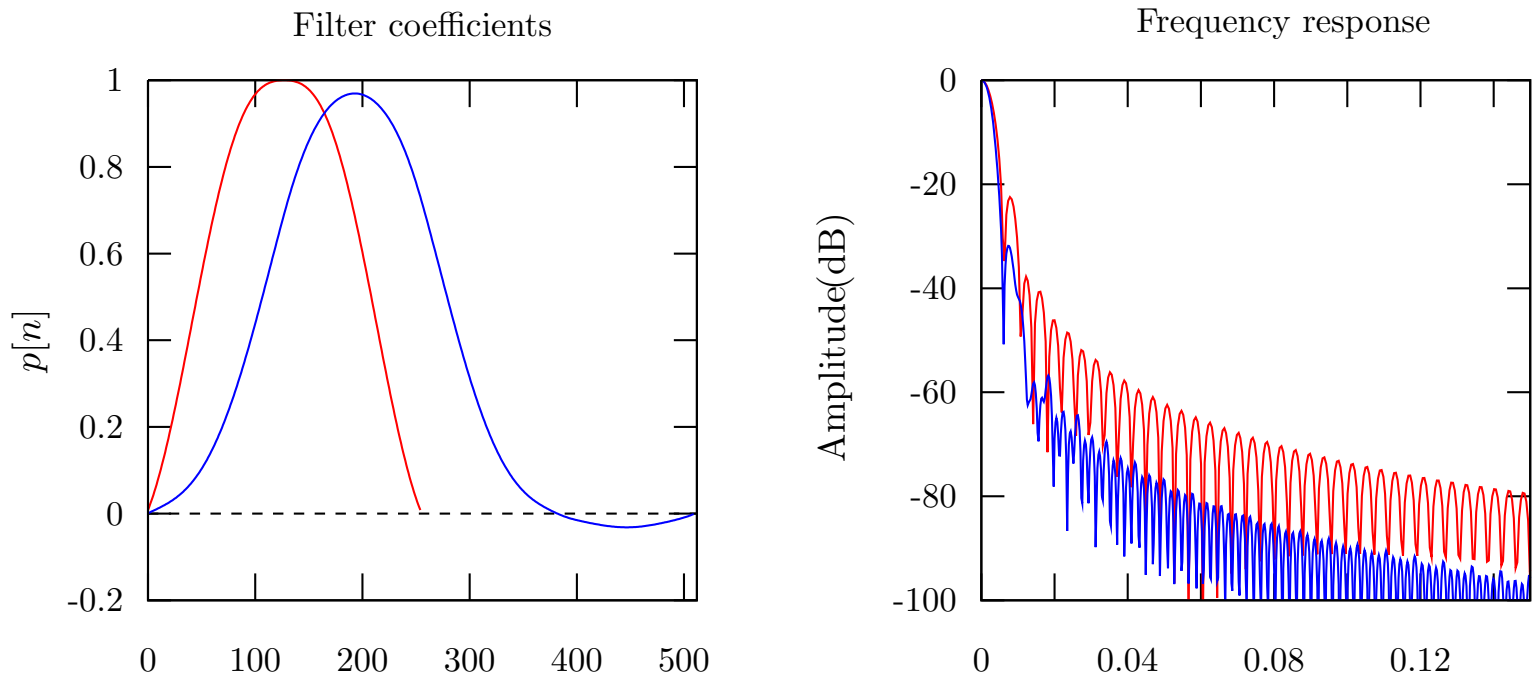

Figure 7: Comparison of filters with length $L=N$ or $L=2 N$ and optimized TFL when $M=128, N=255$ (in red $L=255$ and $\xi=0.9055301$, in blue $L=510$ and $\xi=0.9738691$ ). 
reduced, $\rho \simeq 0.070$. Naturally, then the TFL measures are not as good as in Fig. 7. But, again, one can notice that doubling the length permits a significative improvement and that both windows (for $L=N$ or $2 N$ ) clearly outperform the CP-OFDM TFL which is equal to 0.1088864 and 0.0270732 for $M=128$ and 2048, respectively. Note also that, $\rho$ being given, the TFL for FMT systems decreases as $M$ increases, not to 0 as for CP-OFDM but to the limit $a_{0}\left(\frac{9}{128}\right)$ which is nearly attained for $M=2048$.

Filter coefficients

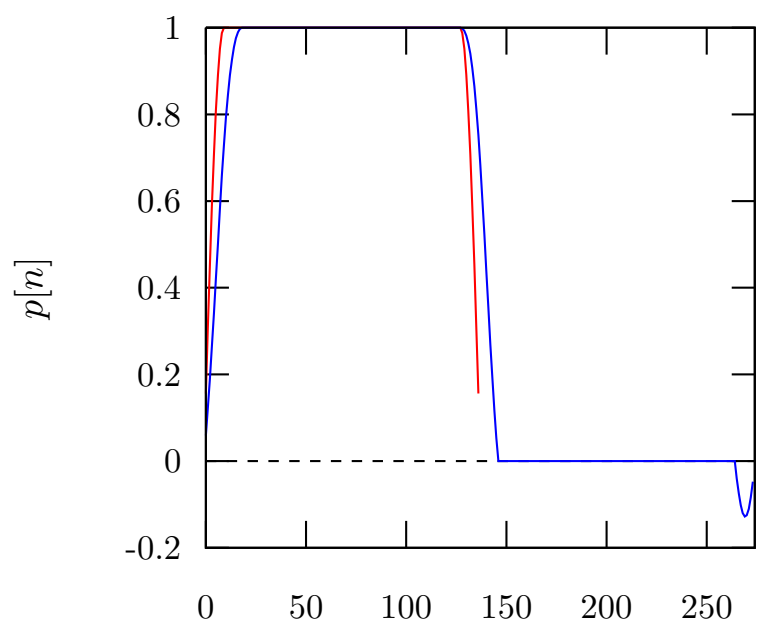

Frequency response

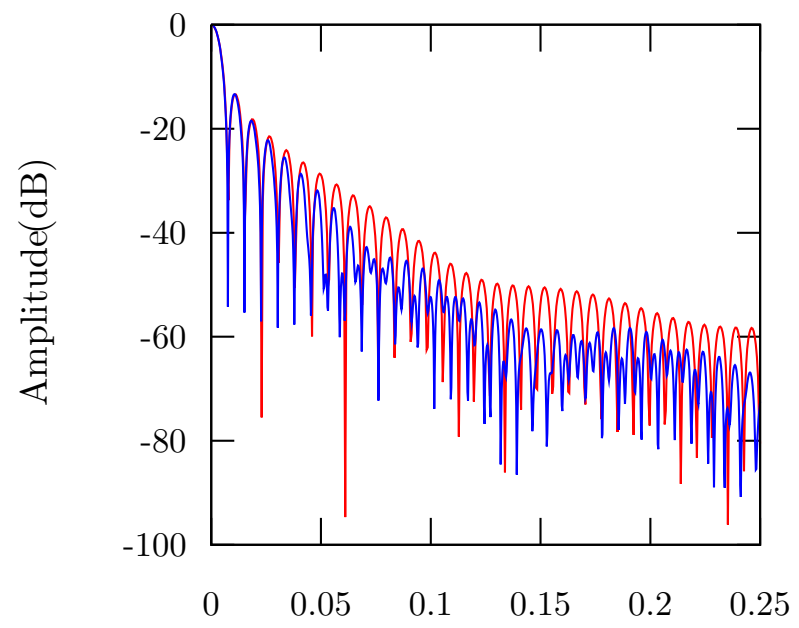

Figure 8: Comparison of filters with length $L=N$ or $L=2 N$ and optimized TFL when $M=128, N=137$ (in red $L=137$ and $\xi=0.3081945$, in blue $L=274$ and $\xi=0.4016572$ ).

\subsection{Arithmetic complexity}

Typically efficient implementations of exponentially modulated filterbank systems, including naturally the FMT ones, involve at the transmitter side an IFFT stage followed by a filtering stage.

As already illustrated with the general algorithm and also in Figures 8 and 9 , if $\rho \leq \frac{1}{2}$ several impulse response coefficients are equal to 1 or 0 . When $\rho \geq \frac{1}{2}$, though less noticeable, some filter coefficients (at least one when $N=2 M-1$ ) are also equal to 0 . Therefore, the computational complexity of the filtering stage can be reduced accordingly.

In Table 4 we display, in terms or real multiplication (RM), the computational complexities for 4 variants of FMT systems of length $L=N$ and $L=2 N$. It is considered in here that, as the $M$-length IFFT complexity in terms of complex multiplications is generally given equal to $\frac{M}{2} \log _{2} M$, its RM cost is equal to $2 M \log _{2} M$.

For the $N$-length FMT system, the extra complexity comes from the $N-M$ coefficients located at the border of the CP-OFDM rectangular window (cf. e.g. [6], [3]). When $L=2 N$ three possibilities are described corresponding to PR FMT systems with $(2 N \leq 3 M)$ or $(2 N \geq 3 M)$ and to a Non PR (NPR) FMT system, that could use for instance a RRC prototype filter. For all systems we assume a transversal form implementation for the filtering stage. 
Filter coefficients

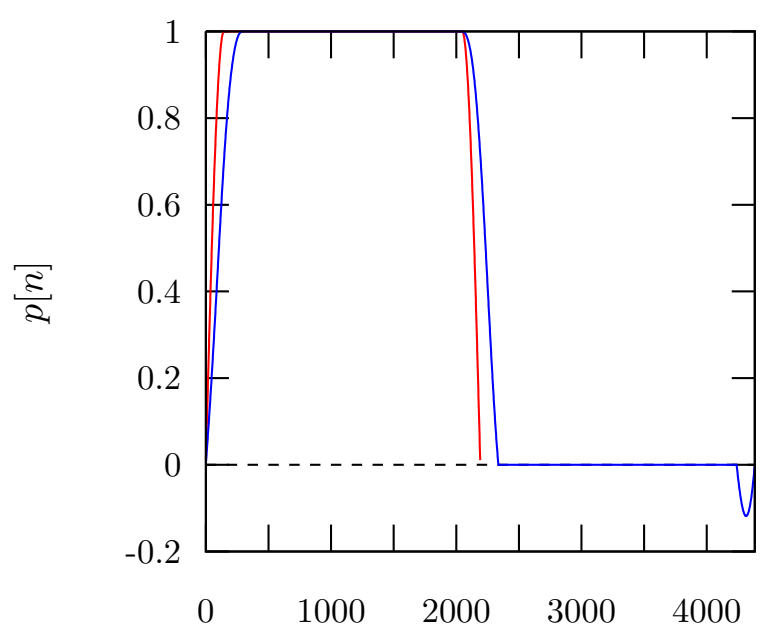

Frequency response

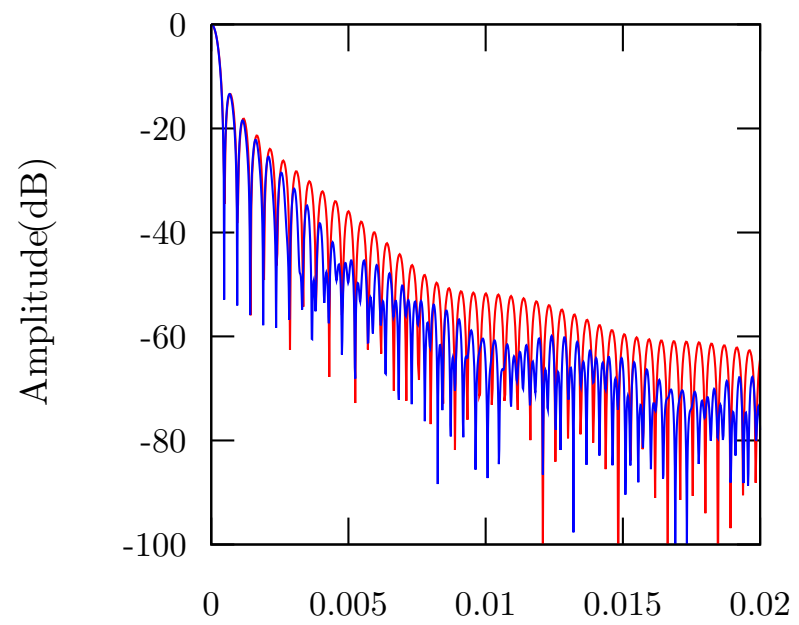

Figure 9: Comparison of filters with length $N$ or $2 N$ and optimized TFL when $M=$ 2048, $N=2192$ (in red $L=2192$ and $\xi=0.2929856$, in blue $L=4384$ and $\xi=0.3941396$ ).

\begin{tabular}{|c|c|c|c|}
\hline$L=N$ & $L=2 N(2 N \leq 3 M)$ & $L=2 N(2 N \geq 3 M)$ & $L=2 N(\mathrm{NPR})$ \\
\hline $2 M \log _{2} M+4(N-M)$ & $2 M \log _{2} M+10(N-M)$ & $2 M \log _{2} M+6 N-4 M$ & $2 M \log _{2} M+4 N$ \\
\hline
\end{tabular}

Table 4: Arithmetic complexity (number of real multiplications) for 4 different variants of the FMT transmitter. 


\begin{tabular}{|c|c|c|c|c|c|}
\hline Syst. & $M$ & $N$ & $L=N$ & $L=2 N$ (PR case) & $L=2 N$ (NPR case) \\
\hline LTE & 128 & 137 & 1.020 & 1.050 & 1.306 \\
\hline LTE & 256 & 274 & 1.018 & 1.044 & 1.268 \\
\hline LTE & 512 & 548 & 1.016 & 1.039 & 1.234 \\
\hline LTE & 1024 & 1096 & 1.014 & 1.035 & 1.214 \\
\hline LTE & 2048 & 2192 & 1.013 & 1.032 & 1.195 \\
\hline P1901 & 8192 & 10296 & 1.040 & 1.099 & 1.193 \\
\hline DVB-T2 & 32768 & 33792 & 1.004 & 1.010 & 1.137 \\
\hline
\end{tabular}

Table 5: Complexity ratios of various FMT systems vs. CP-OFDM.

Naturally, in practice, in order to avoid a large overhead, the redundancy of MC systems is chosen such that $\rho \leq \frac{1}{2}$. Consequently, for practical applications, our PR FMT solutions offer a significant advantage in terms of arithmetic complexity. Furthermore, as Table 5 shows, when taking CP-OFDM as the reference the complexity ratios for PR FMT systems most often induce a moderate extra cost, being at most of $5 \%$ for all the considered systems. Note finally that, as the prototype filter coefficients also involve angular functions, another implementation alternative could be based on a lattice structure.

\section{Conclusion}

A good time-frequency localization is a desirable property for transmission systems that are faced to time and frequency impairments. Unfortunately, CP-OFDM, the most popular multicarrier scheme, behaves badly in the frequency domain. On another hand, the low complexity and latency of OFDM still remain attractive features when designing new systems $[1],[2],[6],[7]$.

In this paper we have presented a design method for a $M$-subcarrier FMT system oversampled by a factor $\frac{N}{M}$ and equipped by prototype filters the length $L$ of which is limited to two CP-OFDM symbols, i.e. $L=2 N$. These FMT systems ensure a perfect reconstruction (PR) property and they are nearly optimal with respect to the Time-Frequency Localization (TFL) criterion. A first key ingredient of the proposed method is a manipulation of the PR equations that takes into account the TFL criterion. By the way, we are able to exhibit a new family of PR FMT solutions that only depends on $2(N-M)$ parameters, instead of $2 N$ using a brute force method.

As, furthermore, we observed that these angular parameters have a regular behavior, we could use a second ingredient consisting in the adaptation to this new problem of the CR introduced in [17]. A few CRs of degree $d$ have been compared and it appeared that we were able to get nearly optimal results with $d \leq 5$ whatever the $N$ and $M$ values in the range $M<N \leq 2 M-1$. Some comparisons with TFL optimization results obtained for $L=N$ [3] have also shown that with $L=2 N$ we could get significantly better localization measures.

For a particular instance of these CRs, i.e. an appropriate selection of the angular parameters values, we could derived simple closed-form expression for a prototype filter we named CF2N. CF2N is for $L=2 N$ the counterpart of the dual RRC proposed in [3] for 
$L=N$. We have shown that when $2 N \leq 3 M$, i.e. the most common case in practice for MC systems, CF2N has TFL performances close to ones of the optimized TFL prototype filters.

Various numerical and graphical results have been presented corresponding to FMT systems with $(N, M)$ parameters values typical of wired (IEEE P1901) and wireless (LTE, DVB-T2) communication standards. Showing that our method could solve design problems of huge size, i. e. hundreds and even thousands of variables.

Finally, we provided a computational complexity analysis that clearly shows the interest of using a PR realization scheme. Indeed, for a bunch of practical examples, it appeared that the extra computational cost compared to the CP-OFDM one does not exceed $5 \%$ while providing a significantly better TFL. 


\section{References}

[1] Qualcomm Technologies. 5G Waveform and multiple access techniques, 2015.

[2] R. Zayani, Y. Medjahdi, H. Shaïek, and D. Roviras. WOLA-OFDM: a potential candidate for asynchronous 5G. In GLOBECOM 2016, Washington, USA, Dec. 2016.

[3] P. Siohan and D. Pinchon. Design of Optimal Short OFDM-Type Windows. Submitted to Signal Processing, 2019.

[4] B. Le Floch, M. Alard, and C. Berrou. Coded Orthogonal Frequency Division Multiplex. Proceedings of the IEEE, 83:982-996, Jun. 1995.

[5] G. Cherubini, E. Eleftheriou, and S. Olcer. Filtered multitone modulation for VDSL. In Proc. of IEEE Global Telecommunications Conference, pages 1139-1144, 1999.

[6] D. Roque and C. Siclet. Performances of Weighted Cyclic Prefix OFDM with low complexity equalization. IEEE Communications Letters, 17(3):439-442, 2013.

[7] Z. Zhao, M. Schellmann, X. Gong, Q. Wang, R. Böhnke, and Y. Guo. Pulse shaping design for OFDM systems. EURASIP Journal on Wireless Communications and Networking, 2017(1):1-25, Apr. 2017.

[8] N. Moret and A.M. Tonello. Design of Orthogonal Filtered Multitone Modulation Systems and Comparison among Efficient Realizations. EURASIP J. Appl. Sig. Proc., pages 1-18, 2010. Article ID 141865.

[9] D. Pinchon and P. Siohan. Closed form expression of optimal short PR FMT prototype filters. In Proceedings Globecom'11 (Houston, USA), Dec. 2011.

[10] D. Pinchon and P. Siohan. Derivation of analytical expressions for flexible PR low complexity FBMC systems. In Proc. EUSIPCO 2013, Marrakech, Morocco, Sep. 2013.

[11] L. Vangelista, N. Benvenuto et al. Key Technologies for Next-Generation Terrestrial Digital Television Standard DVB-T2. IEEE Comm. Magazine, pages 146-153, 2009.

[12] IEEE Standard for Broadband over Power Line Networks: Medium Access Control and Physical Layer Specifications. Dec. 2010.

[13] P. Siohan and M. Renfors. Orthogonal Communication Waveforms. In M. Renfors, X. Mestre, E. Kofidis, and F. Bader, editors, Orthogonal Waveforms and Filter Banks for Future Communication Systems, pages 129-156. Elsevier, 2017.

[14] R. Hleiss, P. Duhamel, and M. Charbit. Oversampled OFDM systems. In Proc. International Conference on Digital Signal Processing, Santorini, Greece, July 1997.

[15] M.I. Doroslovački. Product of second moments in time and frequency for discrete-time signals and the uncertainty limit. Signal Processing, 67(1):59-76, 1998. 
[16] C. Lawrence, J.L. Zhou, and A.L. Tits. User's guide for CFSQP version 2.5: A C code for solving (large scale) constrained nonlinear (minimax) optimization problems, generating iterates satisfying all inequality constraints. Technical Report TR-94-16r1, University of Maryland, 1998.

[17] D. Pinchon, P. Siohan, and C. Siclet. Design techniques for orthogonal modulated filter banks based on a compact representation. IEEE Transactions on Signal Processing, 52(6):1682-1692, June 2004.

[18] C. Brezinski and M. Redivo Zaglia. Extrapolation methods. Theory and practice. NorthHolland, 1991. 